\title{
An Efficient Continuous Hydrothermal Flow Synthesis of Carbon Quantum Dots from a Targeted Biomass Precursor for On-Off Metal Ions Nano- Sensing
}

Ioan-Alexandru Baragau, ${ }^{a}$ Nicholas P. Power, ${ }^{b}$ David J. Morgan, ${ }^{c}$ Richard A. Lobo, ${ }^{d}$ Christopher S. Roberts, ${ }^{\mathrm{e}}$ Maria-Magdalena Titirici, ${ }^{\mathrm{d}}$ Vesna Middelkoop, ${ }^{\mathrm{f}}$ Adriana Diaz, ${ }^{\mathrm{g}}$ Steven Dunn ${ }^{\mathrm{a}}$ and Suela Kellici $^{\mathrm{a}^{*}}$

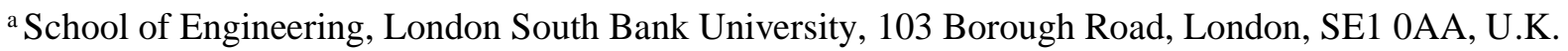
*E-mail: kellicis@1sbu.ac.uk, Website: www.nano2d.co.uk

${ }^{\mathrm{b}}$ School of Life Health \& Chemical Sciences, The Open University, Walton Hall, Milton Keynes, MK7 6AA, U.K.

${ }^{\mathrm{C}}$ Cardiff Catalysis Institute, School of Chemistry, Cardiff University, Park Place, Cardiff, CF10 3AT, U.K.

${ }^{\mathrm{d}}$ Department of Chemical Engineering, Imperial College, South Kensington, London, SW7 2AZ, UK

${ }^{\mathrm{e}}$ Department of Chemistry, Imperial College, South Kensington, London, SW7 2AZ, U.K.

${ }_{\mathrm{f}}^{\mathrm{f}}$ Flemish Institute for Technological Research -VITO, Boeretang 200, 2400 Mol, Belgium

g Ecodesign, Schwindgasse 4/2 1040 Vienna, Austria

Number of pages: 3

Number of figures: 3

Number of tables: 1 


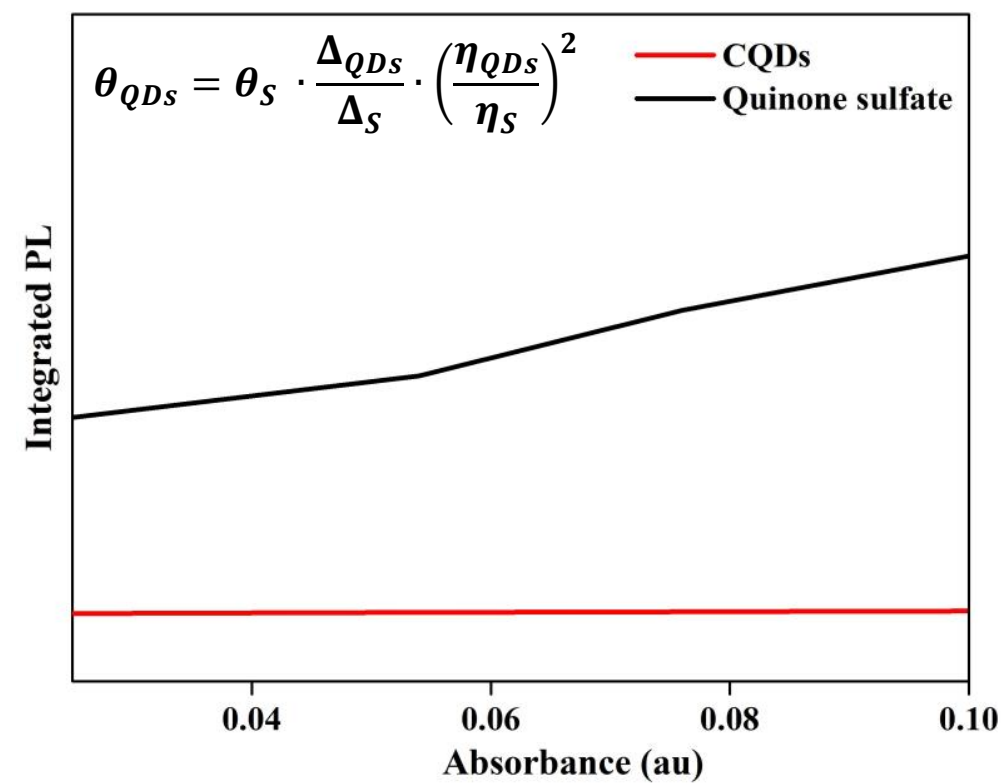

Figure S1: Quantum yield determination via integrated fluorescence intensity vs absorbance plot method.

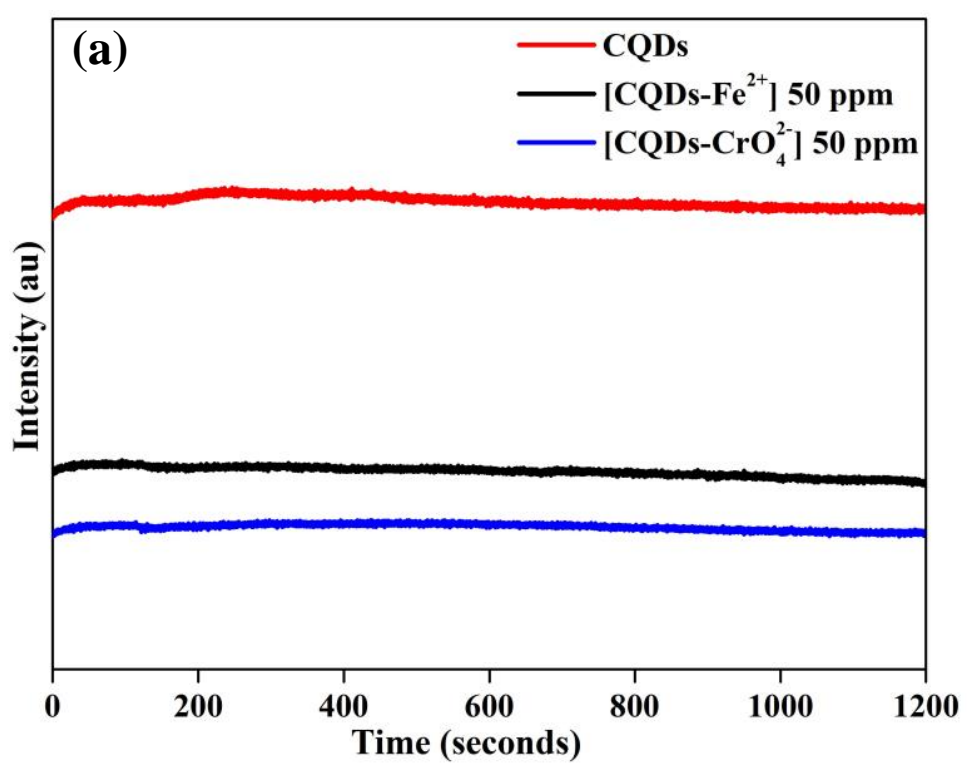

Figure S2: Stability analysis of the CQDs in presence of Cr (VI) (50 ppm) and Fe (II) were made by recording the fluorescence intensity at $446 \mathrm{~nm}$ emission wavelength of the mixture exposed continuously for 1200 seconds (20 minutes) at $360 \mathrm{~nm}$ excitation. 


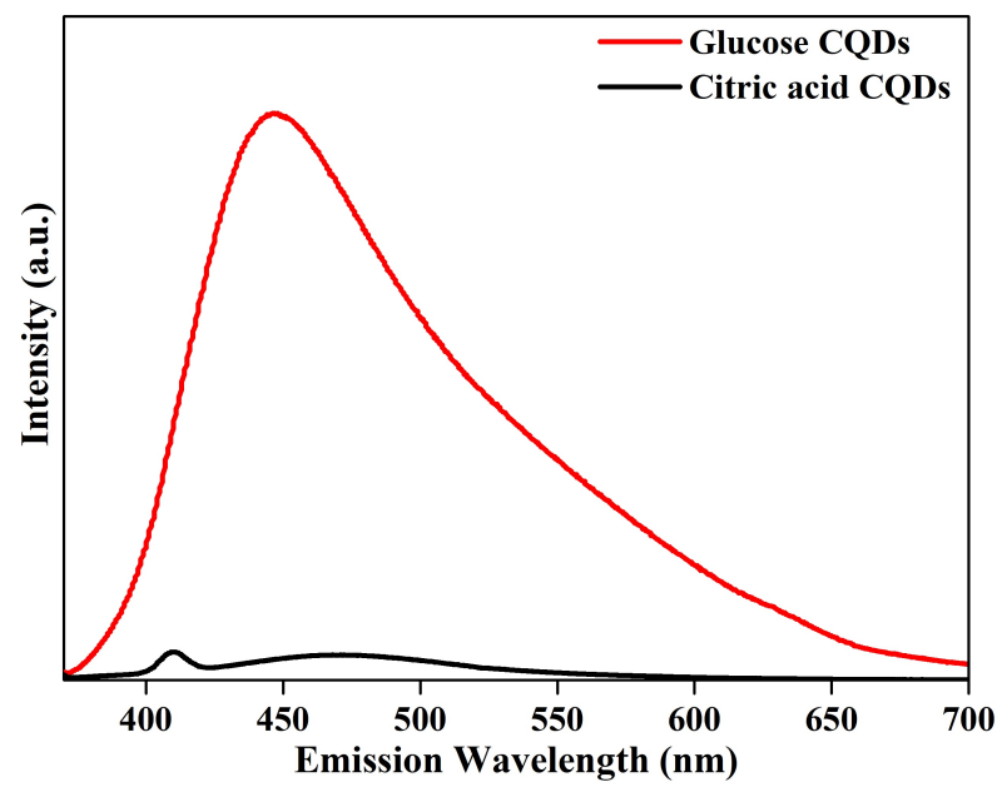

Figure S3: PL spectra of carbon quantum dots synthesised in CHFS using (red line) glucose and (black line) citric acid as carbon precursors.

Table S1: Experimental and inventory data (for glucose, water and electricity consumption values) utilised in LCA analysis.

\begin{tabular}{|c|c|c|c|c|c|}
\hline Inventory data & Unit & \multicolumn{2}{|c|}{ Batch synthesis } & \multicolumn{2}{|l|}{ CHFS } \\
\hline $\begin{array}{l}\text { Glucose (via starch hydrolysis } \\
\text { from corn) }\end{array}$ & g & & 120 & & 14 \\
\hline $\begin{array}{l}\text { Water, deionised \{Europe without } \\
\text { Switzerland\} | market for water, } \\
\text { deionised | Cut-off, } U\end{array}$ & g & & 600 & & 1186 \\
\hline $\begin{array}{l}\text { Electricity, low voltage }\{R E R\} \mid \\
\text { market group for | Cut-off, } U\end{array}$ & kWh & NA & & $\begin{array}{l}2 \text { pumps of } 60 \mathrm{~W}, 94 \\
\min =1.57 \mathrm{hrs}\end{array}$ & 0.08 \\
\hline $\begin{array}{l}\text { Electricity, low voltage }\{\text { RER\}| } \\
\text { market group for | Cut-off, } U\end{array}$ & kWh & $\begin{array}{l}\text { heater } 1000 \mathrm{~W} \\
6 \mathrm{~h}, 200^{\circ} \mathrm{C}\end{array}$ & 6 & $\begin{array}{c}\text { heater } 1000 \mathrm{~W}, \\
94 \mathrm{~min}=1.57 \mathrm{hrs}, \\
450^{\circ} \mathrm{C}\end{array}$ & 0.6666 \\
\hline
\end{tabular}

